OPEN ACCESS

Edited by:

Kai Lu,

Fujian Agriculture and Forestry

University, China

Reviewed by:

Shunhua Gui,

Ghent University, Belgium

Honggang Tian,

Northwest A\&F University, China

Hamzeh Izadi,

Vali-E-Asr University of Rafsanjan, Iran

*Correspondence:

Can $\mathrm{Li}$

lican790108@163.com

${ }^{t}$ These authors have contributed

equally to this work

Specialty section:

This article was submitted to Invertebrate Physiology, a section of the journal

Frontiers in Physiology

Received: 23 June 2020 Accepted: 04 August 2020

Published: 20 August 2020

Citation:

Xu K-K, Pan B-Y, Wang Y-Y,

Ren Q-Q and Li C (2020) Roles of the PTP61F Gene in Regulating Energy Metabolism of Tribolium castaneum

(Coleoptera: Tenebrionidae).

Front. Physiol. 11:1071

doi: 10.3389/fphys.2020.01071

\section{Roles of the PTP61F Gene in Regulating Energy Metabolism of Tribolium castaneum (Coleoptera: Tenebrionidae)}

\author{
Kang-Kang Xu't , Bi-Ying Pan ${ }^{2 t}$, Yuan-Yuan Wang ${ }^{1}$, Qian-Qian Ren ${ }^{1}$ and Can Li ${ }^{1 *}$
}

${ }^{1}$ Guizhou Provincial Key Laboratory for Rare Animal and Economic Insect of the Mountainous Region, Guizhou Provincial Engineering Research Center for Biological Resources Protection and Efficient Utilization of the Mountainous Region, College of Biology and Environmental Engineering, Guiyang University, Guiyang, China, ${ }^{2}$ College of Life and Environmental Sciences, Hangzhou Normal University, Hangzhou, China

Protein tyrosine phosphatase 1B (PTP1B) is a negative regulator in the insulin signaling pathway. It belongs to a class of non-receptor phosphatases of protein tyrosine phosphatase and can catalyze the dephosphorylation of tyrosine to regulate cell differentiation, growth, and metabolism. However, few studies have focused on the role of PTP1B in regulating energy metabolism of insects. In this study, we investigated the expression profiles and the functions of a PTP1B gene (designated TCPTP61F) in the red flour beetle Tribolium castaneum. Quantitative real-time PCR analyzed showed that TCPTP61F was highly expressed in the pupal and adult stages. In adult tissues, TCPTP61F was prominently expressed in the tarsus and head. RNA interferencemediated silencing of TCPTP61F reduced the expression of eight genes in trehalose metabolic and glycolytic pathways. TCPTP61F depletion also caused a significant change in the distribution of trehalose, glucose, and glycogen. Additionally, knockdown of TCPTP61F inhibited the pyruvate kinase (PK) activity and significantly decreased the adenosine triphosphate (ATP) level. The results suggest that TCPTP61F is indispensible for trehalose and energy metabolism of $T$. castaneum.

Keywords: Tribolium castaneum, protein tyrosine phosphatase, RNA interference, trehalose metabolism, energy metabolism

\section{INTRODUCTION}

Insects need a continuous supply of energy to maintain their metabolism and activity. Energy metabolism in the insect body is constant. Insects directly use trehalose as their main energy source and it serves as the main sugar component of insect hemolymph (Yasugi et al., 2017). Trehalose is a highly stable, non-reducing disaccharide formed by two glucose molecules. It is found in a variety of organisms including bacteria, yeast, fungi, nematodes, insects, and some other invertebrates, but not in mammals (Elbein et al., 2003). In addition to playing a crucial role as an immediate source of energy, trehalose also plays important functions in insect response to stresses such as high or low temperatures, poor nutrition or starvation, oxidation, high osmotic pressure, toxic substances, and UV-B irradiation (Tamang et al., 2017; Chen J. X. et al., 2018). Under the catalysis of trehalose phosphate synthase (TPS), uridine diphosphate (UDP) glucose (UDP-glucose) and glucose-6-phosphate (G-6-P) synthesize trehalose-6-phosphate, which is then dephosphorylated 
under the action of 6-trehalose phosphate esterase (TPP) to produce trehalose. This is the most important path of trehalose synthesis in insects (Shukla et al., 2015; Tang et al., 2017; Chen J. X. et al., 2018).

When energy is needed, trehalose is hydrolyzed into glucose under the catalysis of trehalase (TRE), the only enzyme known to irreversibly degrade trehalose (Barraza and Sánchez, 2013). Glucose then enters the glycolysis-tricarboxylic acid (glycolysisTCA) cycle. In the glycolysis pathway, glucose serves as the initial substrate and is converted into pyruvate by a series of enzymes, including hexokinase ( $\mathrm{HK})$, glucose-6-phosphate isomerase (G6PI), phosphofructokinase (PFK), and pyruvate kinase (PK) (Hu et al., 2016). Pyruvate can be further converted into acetyl-coenzyme A (acetyl-CoA), which can combine with oxaloacetic acid to enter TCA cycle and finally be oxidized to $\mathrm{CO}_{2}, \mathrm{H}_{2} \mathrm{O}$, and adenosine triphosphate (ATP) (Hu et al., 2016). Insects could adapt to various physiological activities by regulating the rate of the glycolysis-TCA cycle. For example, some insects reduce the expression of glycolysis or TCA metabolic enzyme genes in the early pupal stage which saves energy for the development of new organs. These species include Drosophila melanogaster, Bombyx mori, and Spodoptera litura (White et al., 1999; Tian et al., 2010; Hu et al., 2016).

Insulin is important in regulating glucose homeostasis, lipid metabolism, and energy balance (White, 1998; Czech and Corvera, 1999). It can increase the transport of glucose and the synthesis of glycogen, diminish gluconeogenesis, inhibit glycogenolysis, and regulate the expression of many genes (Lochhead et al., 2001; Vital et al., 2010). Components of the insulin signaling pathway are extremely conserved in organisms as distantly related as humans, D. melanogaster, and Caenorhabditis elegans (Leevers, 2001). In Aedes aegypti, upstream components of the insulin signaling pathway, such as phosphatidylinositol 3-kinase (PI3K) (Pri-Tal et al., 2008) and protein kinase B (AKT) (Riehle and Brown, 2003), have been associated with glucose metabolism. The insulin signaling pathway not only controls the metabolic balance of blood glucose but also directly regulates juvenile hormones and ecdysone, which control insect development, metamorphosis, and reproduction (Satake et al., 1997; Vital et al., 2010; Kim and Hong, 2015).

Protein tyrosine phosphatases (PTPs, EC 3.1.3.48) are a large family of enzymes that regulate insulin signal transduction and are also involved in cell signal transduction and cell cycles (Klaman et al., 2000; Tonks, 2006). The PTP family is composed of four different subfamilies, classes I, II, III and IV (Sacco et al., 2012). Class I Cys-PTPs are the largest group of PTPs and could be divided into "classical" and dual specificity phosphatases, in which classical phosphatases are strictly devoted to the dephosphorylation of phosphotyrosine residues (Alonso et al., 2004). The family genes additionally participate in other physiological activities and metabolic processes such as cell differentiation, transformation, growth, reproduction, and immunity (Abaskharoun et al., 2010; Bao et al., 2011; Alho et al., 2013; Agouni et al., 2014). Protein tyrosine phosphatase $1 \mathrm{~B}$ (PTP1B) is involved in the regulation of insulin action and other signal transduction pathways. PTP1B is a major regulator of energy balance, insulin sensitivity, and fat storage in insect body. In Locusta migratoria tibetensis, PTPN1-encoded insulin receptor inhibitor tyrosine protein phosphatase non-receptor type PTP1B is a negative regulator of the insulin pathway, and it plays an important role in response to hypoxic stress (Ding et al., 2018). The homologous gene of $P T P 1 B$ in D. melanogaster encoded by PTP61F specifically targets to Dock and enhances the signal selectivity of insulin receptor (Wu et al., 2011).

The red flour beetle, Tribolium castaneum (Herbst) (Coleoptera: Tenebrionidae), is a worldwide pest of stored grains (Mehmood et al., 2018; Chen Q. W. et al., 2018). It has glands that secrete a liquid which causes a moldy smell in flour and this secretion also contains the carcinogen benzoquinone (Boateng and Obengofori, 2008). Control of T. castaneum in stored products and grain is primarily by fumigants and sprays, but insecticide resistance is now a major problem (Pimentel et al., 2010; Perkin and Oppert, 2019). Most importantly, T. castaneum is a model insect often used for research on gene function (Lorenzen et al., 2007). To date, few studies have evaluated the role of PTP1B in regulating energy metabolism in insects. In this present study, we identified and obtained a $P T P 1 B$ gene (TcPTP61F) from T. castaneum and analyzed its expression patterns in different developmental stages and tissues. We used RNA interference in vivo to efficiently disrupt the TcPTP61F gene function in order to clarify its role in regulating the energy metabolism of T. castaneum.

\section{MATERIALS AND METHODS}

\section{Insect and Sample Preparation}

The laboratory stock colony of T. castaneum were raised on whole wheat flour containing $5 \%$ yeast in an incubator at $28 \pm 1^{\circ} \mathrm{C}$ and $65 \pm 5 \%$ relative humidity under a constant $24 \mathrm{~h}$ dark (0L:24D).

In the tissue-specific experiment, the adults (male to female ratio 1:1) were used for tissue dissection. The antennae, head, wing, tarsus, epidermis, midgut, and fat body of T. castaneum were dissected under a stereomicroscope (Olympus SZX12, Tokyo, Japan). Pools of 200 individuals were used to prepare each body part. Samples at different developmental stages, including early larvae (EL, 2nd instar), middle larvae (ML, 5th instar), and late larvae (LL, 8th instar); early pupae (EP, 1st day), middle pupae (MP, 3rd day), and late pupae (LP, 5th day); and early adults (EA, 1st day), middle adults (MA, 3rd day), and late adults (LA, 5th day) were collected separately. T. castaneum used for the microinjection of double-stranded RNA (dsRNA) were the 1st day of the 8th instar larvae, and the insects at 48 and $72 \mathrm{~h}$ after injection were collected for determination of gene expression, carbohydrate content, and ATP content. Ten individuals were used for gene expression, and 20 for carbohydrate, and ATP contents, respectively. All above the samples were immediately frozen in liquid nitrogen and stored at $-80^{\circ} \mathrm{C}$. All of the experiments were repeated three times.

\section{RNA Isolation and cDNA Synthesis}

Total RNA was isolated from each sample by using the MiniBEST Universal Extraction Kit (TaKaRa, Dalian, China), 
following the manufacturer's instructions. Total RNA integrity was evaluated using $1 \%$ agarose gel electrophoresis, and the RNA concentration and purity were determined by a NanoDrop 2000C Spectrophotometer (Thermo Fisher Scientific, Waltham, MA, United States). First-strand complementary DNA (cDNA) synthesis was performed using the PrimeScript ${ }^{\circledR}$ RT Reagent Kit (TaKaRa, Dalian, China) following manufacturer's instructions.

\section{Synthesis and Injection of dsRNA}

RNAi was used to study the potential function of TcPTP61F in T. castaneum. The dsRNA primers (Table 1) for TcPTP61F and green fluorescent protein (GFP, as control) were designed using E-RNAi ${ }^{1}$. The PCR products were subject to $\mathrm{T}$ cloning, followed by a subsequent amplification with primers containing the T7 promoter sequence. Cross-PCR reactions were performed using a T7 RiboMAX ${ }^{\mathrm{TM}}$ Express RNAi System kit (Promega, Madison, WI, United States) to synthesize dsRNA. The dsRNA was diluted with nuclease-free water to a final concentration of $2 \mu \mathrm{g} / \mu \mathrm{L}$. Using a Nanoliter 2010 injector (World Precision Instruments, Sarasota, FL, United States), $200 \mathrm{ng}$ of dsTcPTP61F or dsGFP was slowly injected into the hemocoel between the third and fourth abdominal segments of each 1 st day of the 8th instar larvae. Three biological replicates (each with at least 50 larvae) were treated by dsTcPTP61F and dsGFP injection. The insects treated with dsRNA were reared under the same conditions as mentioned above.

\section{Quantitative Real-Time Polymerase Chain Reaction (qPCR)}

The qPCR was conducted to confirm the relative expression levels of PTP61F in various developmental phases and tissues. At 48 and $72 \mathrm{~h}$ after dsRNA injection, the insects from each treatment were collected for qPCR to assess the efficiency of the RNAi. After PTP61F was knocked down, transcript levels of six trehalose metabolic pathway genes, including five trehalases (TcTre1-1, TcTre1-2, TcTre1-3, TcTre1-4, and TcTre2) and trehalose-6-phosphate synthase gene (TcTPS); and glycolytic pathway genes, such as hexokinases (TcHK1 and TcHK2) and glucose-6-phosphate isomerase (TcG6PI); and pyruvate kinase gene $(T c P K)$ were detected by qPCR after injection. The qPCR was carried out on a CFX-96 real-time detection system (Bio-Rad, Hercules, CA, United States) in a $20 \mu \mathrm{L}$ reaction containing $1 \mu \mathrm{L}(100 \mathrm{ng} / \mu \mathrm{L})$ cDNA, $1 \mu \mathrm{L}(10 \mu \mathrm{M})$ each primer (Table 1), $7 \mu \mathrm{L}$ nuclease-free water, and $10 \mu \mathrm{L}$ of GoTaq $^{\circledR}$ qPCR MasterMix (Promega). The reaction was performed under the following conditions: pre-incubation at $95^{\circ} \mathrm{C}$ for $2 \mathrm{~min}$, followed by 40 cycles of $95^{\circ} \mathrm{C}$ for $30 \mathrm{~s}$ and annealing at $60^{\circ} \mathrm{C}$ for $30 \mathrm{~s}$, with a melting curve at $65-95^{\circ} \mathrm{C}$. Amplification of Ribosomal Protein L13a (RPL13a) was used as an internal control. All of the experiments were performed in triplicate, with two technical replicates each. The $2^{-\triangle \Delta C T}$ method was used for the analysis of relative gene expression (Livak and Schmittgen, 2001).

\footnotetext{
${ }^{1}$ http://www.dkfz.de/signaling/e-rnai3/idseq.php
}

\section{Analysis of Trehalose, Glucose, and Glycogen Contents}

Twenty individuals, collected after injection, were used to measured trehalose, glucose and glycogen contents. The anthrone-sulfuric acid method was used to measure the trehalose content (Leyva et al., 2008). The assay of glucose and glycogen contents was performed according to the previously described methods (Zhang et al., 2017). Three independent biological replicates were used for assays. Briefly, the samples were homogenized in $200 \mu \mathrm{L}$ phosphate buffer saline (PBS; $\mathrm{pH} 7.0$ ), then $800 \mu \mathrm{L}$ PBS was added up to $1 \mathrm{~mL}$. Subsequently, the homogenate was centrifuged at $1,000 \mathrm{~g}$ for $20 \mathrm{~min}$ at $4^{\circ} \mathrm{C}$. The supernatant $(300 \mu \mathrm{L})$ was taken to detect concentrations of protein, trehalose, and glycogen as described below. Then $350 \mu \mathrm{L}$ of the supernatant was removed and ultracentrifuged at 20,800 g for $60 \mathrm{~min}$ at $4^{\circ} \mathrm{C}$. The supernatant $(300 \mu \mathrm{L})$ obtained from ultracentrifugation was used to determine the concentration of protein and glucose. The sediment was suspended in PBS (300 $\mu \mathrm{L}$ ) then used for the determination of protein and glucose contents. The glucose content was determined using a glucose (GO) Assay Kit (Sigma-Aldrich, St. Louis, MO, United States) according to manufacturer instructions. As for the determination of glycogen content, the methods were similar to the methods of glucose content measurement unless the samples required $4 \mathrm{~h}$ decomposition reaction by amyloglucosidase (catalog no. 10115, Sigma-Aldrich). The protein content was determined using the BCA Protein Assay Kit (Beyotime, China).

\section{Determination of PK Activity and Measurement of ATP Content}

The beetles were mixed into physiological saline $(\mathrm{g}: \mathrm{mL}=1: 9)$ for grinding and crushing to obtain a $10 \%$ homogenate. The $10 \%$ homogenate was prepared with physiological saline, and the experiment was conducted according to the instruction on the Pyruvate Kinase Assay Kit (No. A076-1-1, Nanjing Jiancheng Bioengineering Institute) to determinate the PK activity. Measurement of ATP content was determined according to the instruction of ATP Assay Kit (No. A095-1-1, Nanjing Jiancheng Bioengineering Institute).

\section{Statistical Analyses}

All of the data were presented as mean \pm standard error (SE) and were analyzed using SPSS version 20 software (SPSS Inc., Chicago, IL, United States). A one-way analysis of variance (ANOVA) followed by a least significant difference (LSD) test was used for comparing the differences among more than two samples. Differences between two groups were compared using Student's $t$-test $\left({ }^{*} P<0.05,{ }^{* *} P<0.01\right)$.

\section{RESULTS}

\section{The Developmental and Tissue Expression Profiles of TCPTP61F}

We measured the expression level of TcPTP61F in the antenna, head, wing, tarsus, epidermis, midgut, and fat body from adult 
TABLE 1 | Primers used to synthesize dsRNA and analyze transcript levels.

\begin{tabular}{|c|c|c|c|c|}
\hline Application of primers & Gene name & Forward primer $\left(5^{\prime}-3^{\prime}\right)$ & Reverse primer $\left(5^{\prime}-3^{\prime}\right)$ & Length (bp) \\
\hline \multirow[t]{2}{*}{ dsRNA synthesis } & TCPTP61F & T7-GTCATCGGGCAATAACATC & T7-ATATCTCGGGACTCTITCGT & 584 \\
\hline & GFP & T7-AAGGGCGAGGAGCTGTTCACCG & T7-CAGCAGGACCATGTGATCGCGC & 256 \\
\hline \multirow[t]{12}{*}{ qPCR analysis } & TCPTP61F & CCAAATATCCCCAAGAGC & GACTATCGGAACGCAAATC & 151 \\
\hline & TcTre1-1 & AACGACTCGCAATGGCTGG & CGGAGGCGTAGTGGAATAGAG & 127 \\
\hline & TcTre1-2 & GTGCCCAATGGGTIATCG & CAACCACAACACTTCCTTCG & 261 \\
\hline & TcTre1-3 & ССTCTCATTCGTCACAAGCG & AAGCGTITGATICTITGCG & 205 \\
\hline & TcTre1-4 & ACGGTGCCCGCATCTACTA & GTGTAGGTGGTCCCGTTCTTG & 187 \\
\hline & TcTre2 & CTCAGCCTGGCCCTTAGTTG & GGAGTCCTCGTAGATGCGTT & 120 \\
\hline & TCTPS & CGATTCGTACTACAACGGCTGC & GTGGTGTAGCATTGCCAGTGC & 105 \\
\hline & TCHK1 & CGCACCGAATGCCAGAATC & GACCCACCCGACATCGATT & 141 \\
\hline & TCHK2 & CGAATCGGCCTAATAGTTGGC & GACGGAGCCCTCGATITCAT & 155 \\
\hline & TCG6PI & GTGATGCCGGAGGTGAAT & CACGTCGGTGATGGGCTT & 112 \\
\hline & TCPK & CAATITACCGCATCTCAAC & GTCTCCATCATITCTCCAAC & 240 \\
\hline & TCRPL13a & ACCATATGACCGCAGGAAAC & GGTGAATGGAGCCACTTGTT & 250 \\
\hline
\end{tabular}

T7:GGATCCTAATACGACTCACTATAGG.

T. castaneum to study tissue-specific expression profiles of TcPTP61F (Figure 1A). Among the seven tissues, the highest expression level of TcPTP61F was detected in the tarsus, followed by the head and epidermis; expression was relatively lower in the other tissues. As for developmental expression profiles illustrated in Figure 1B, the mRNA of TCPTP61F was highly expressed in the pupal and adult stages was stable at a low expression level in larvae. The results showed that the expression of TcPTP61F varied across developmental stages and among many tissues.

\section{Evaluation of the Efficiency of RNAi Knockdown by dsTcPTP61F}

To determine the effect of RNAi, the relative expression level of TcPTP61F were detected by qPCR. The results showed that the expression level of TcPTP61F was significantly decreased at 48 and $72 \mathrm{~h}$ after dsTcPTP61F injection $(P<0.01)$ (Figure 2 ), and the interference efficiency were 88.14 and $94.29 \%$, respectively, indicating the successful inhibition of the target genes.

\section{Effects on the Relative Expression Levels of Genes in the Trehalose Metabolic Pathway Following TcPTP61F Knockdown}

The results showed that the mRNA levels of TcTre1-1, TcTre14, TcTre2, and TcTPS decreased significantly at 48 and $72 \mathrm{~h}$ after TcPTP61F was inhibited $(P<0.05)$ (Figures 3A,D-F). The expression levels of TcTre1-2 and TcTre1-3 decreased at $48 \mathrm{~h}$ but increased significantly at $72 \mathrm{~h}$ after injection of dsTcPTP61F $(P<0.01)$ (Figures 3B,C).

\section{Effects on the Content of Trehalose, Glucose, and Glycogen Following dsTcPTP61F Injection}

The content of trehalose increased $52.62 \%$ at $48 \mathrm{~h}$ after injection with dsTcPTP61F $(P<0.01)$ and then was restored to a normal level at $72 \mathrm{~h}$ compared to the dsGFP group (Figure 4A).
In contrast, the glycogen and glucose contents significantly increased at $72 \mathrm{~h}$ after TcPTP61F was knocked down $(P<0.05)$ (Figures 4B,C). The glycogen content was stable at $48 \mathrm{~h}$ after dsTcPTP61F injection (Figure 4B), whereas the glucose content decreased significantly $(P<0.05)$ (Figure 4C).

\section{Effects of TCPTP61F Knockdown on the Relative Expression of Critical Genes in the Glycolytic Pathway}

After TCPTP61F was knocked down, the relative expressions of $H K 1$ and $H K 2$ were significantly downregulated at 48 and $72 \mathrm{~h}$ $(P<0.01)$ (Figures 5A,B). In addition, the relative expression of G6PI was significantly downregulated at $48 \mathrm{~h}$, but upregulated sharply at $72 \mathrm{~h}(P<0.01)$ (Figure 5C).

\section{Effects on the Enzyme Activity of PK and ATP Content After TcPTP61F Knockdown}

When TcPTP61F was inhibited, the enzyme activity of PK significantly decreased at 48 and $72 \mathrm{~h}$, especially it was decreased at $48 \mathrm{~h}$ by $42.03 \%$ compared to the dsGFP group $(P<0.05)$ (Figure 6A). Consistently, the mRNA level of $T c P K$ was significantly down-regulated at 48 and $72 \mathrm{~h}$ after TcPTP61F suppression $(P<0.01)$ (Figure 6B). The ATP content decreased 63.61 and $23.15 \%$ at 48 and $72 \mathrm{~h}$ after dsTcPTP61F injection, respectively (Figure 7 ).

\section{DISCUSSION}

Protein phosphorylation and dephosphorylation is one of the most important biochemical reactions in the body and plays a role in regulating cell growth, proliferation, differentiation, and immunity (Mustelin et al., 2005; Tonks, 2006). Protein tyrosine phosphatase is a type of superfamily phosphatase that changes its phosphate by specifically catalyzing the removal of phosphate groups on phosphorylated modified tyrosine residues to control cell function (Tonks, 2006). 

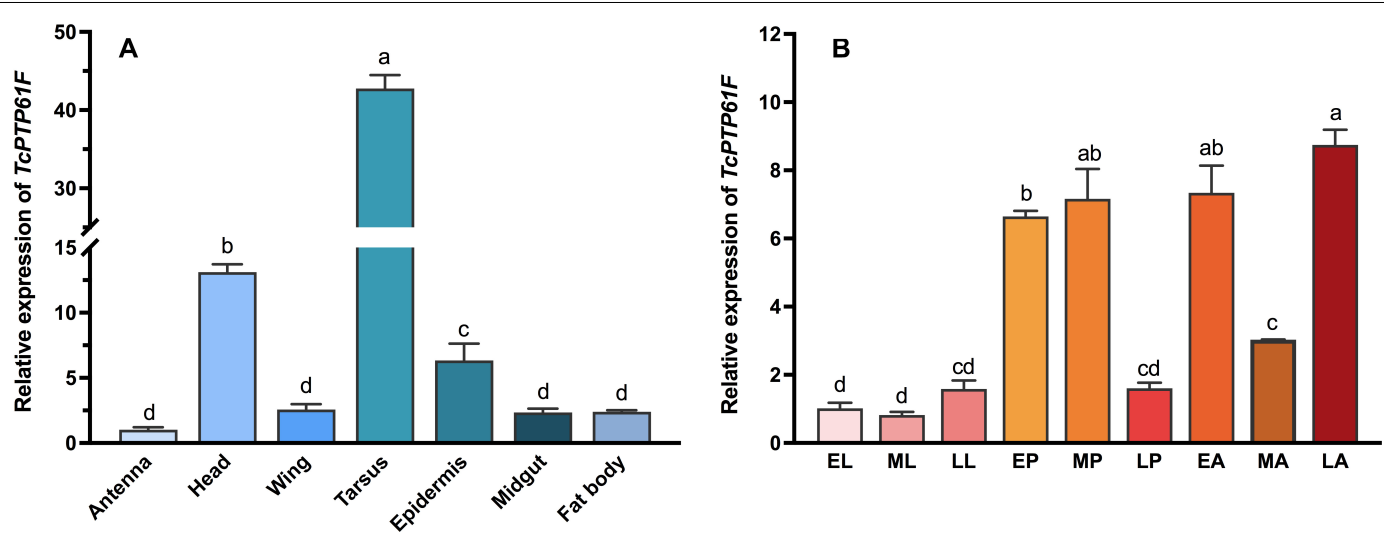

FIGURE 1 | Relative expression levels of TCPTP61F in different tissues (A) and different developmental stages (B) of Tribolium castaneum. EL, ML, LL, EP, MP, LP, EA, MA, and LA represent early instar larvae, middle instar larvae, late instar larvae, early pupae, middle pupae, late pupae, early adults, middle adults, and late adults, respectively. Different letters above bars indicate significant differences based on one-way ANOVA followed by a least significance difference test $(P<0.05)$.

Although the amino acid sequences and substrates of PTPs differ, most PTPs have a conserved structural motif (Motif) (H/V) C (X) R (S/T) (Supplementary Figure S1), and this structural phantom plays a key role in the catalytic activity of PTP (Wang et al., 2014). The amino acid sequences of PTP1B in several insect species contain a catalytic domain of protein tyrosine phosphatase (PTPc) (Supplementary Figure S2).

PTP1B is a ubiquitously expressed intracellular protein tyrosine phosphatase (Forsell et al., 2000). It is common in the testis, kidney, spleen, muscle, heart, liver, and brain of mice (Miyasaka and Li, 1992). However, there are few studies of PTP1B in insects. In A. aegypti, it has been reported to occur in all tissues, and it is highly expressed in the ovaries (Moretti et al., 2014). In this study, we found that TcPTP61F is expressed in a variety of $T$. castaneum tissues. This study did not evaluate the T. castaneum ovary but it showed that TcPTP61F was highly expressed in the tarsus and the head. Muscle has been revealed as one of the insulin's key target tissues (Saltiel and Kahn, 2001; Biddinger and Kahn, 2006), and it is known that tarsus contains a great number of muscle, which may be the main reason of the high expression of TcPTP61F in tarsus. Like the mosquito, the head of $T$. castaneum is also composed of many different structures and tissues, and responsible for several physiological processes (Predel et al., 2010). Meanwhile, phosphorylation of tyrosine residues induces phosphotyrosine (pTyr) signaling pathways are important signal transduction systems for many cellular functions such as protein synthesis and cell proliferation. Further studies are required to clarify the roles of TCPTP61F in the tarsus and head of T. castaneum. In the developmental pattern of TcPTP61F, we found relatively lower expression in the larval stage, but higher expression in the pupal and adult stages, which demonstrates differences in TcPTP61F at different developmental stages. A transient increase occurred in the total tyrosine phosphorylation of the A. aegypti head during the first days after adult emergence (Jablonka et al., 2011), and our results are consistent with the those in A. aegypti.

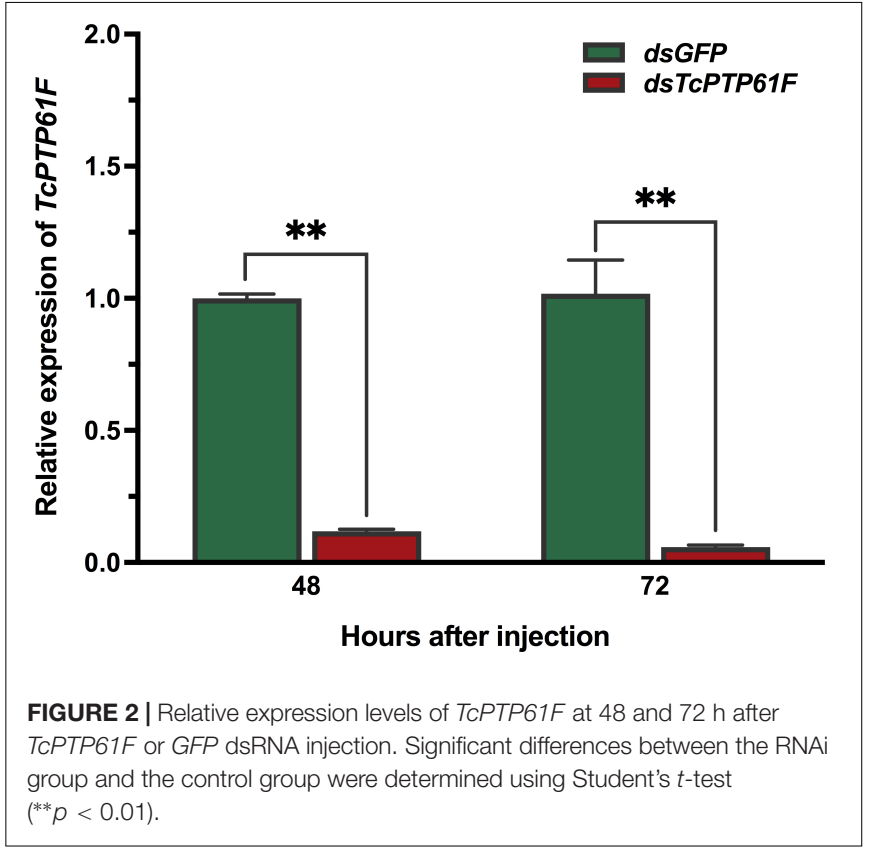

PTP1B is a member of the PTPs family and plays a key regulatory role in insulin and leptin signaling. By catalyzing the dephosphorylation of phosphotyrosine (pTyr), it maintains the level of phosphorylation of protein tyrosine together with protein tyrosine kinase (PTKs) (Tiganis and Bennett, 2007). PTP1B regulates the insulin signaling pathway with tissue specificity. The lack of T-cell protein tyrosine phosphatase (TCPTP) in the muscles of mice does not alter insulin signaling and glucose homeostasis (Loh et al., 2012). RNAi is an effective means to inhibit gene expression through dsRNA injection and it is widely used to study gene function (Prentice et al., 2017). To explore the potential role of TcPTP61F in $T$. castaneum, we knocked down TcPTP61F using RNAi technology. The expression of TcPTP61F decreased significantly at 48 and $72 \mathrm{~h}$ following dsTcPTP61F injection. Trehalose can provide energy to promote 

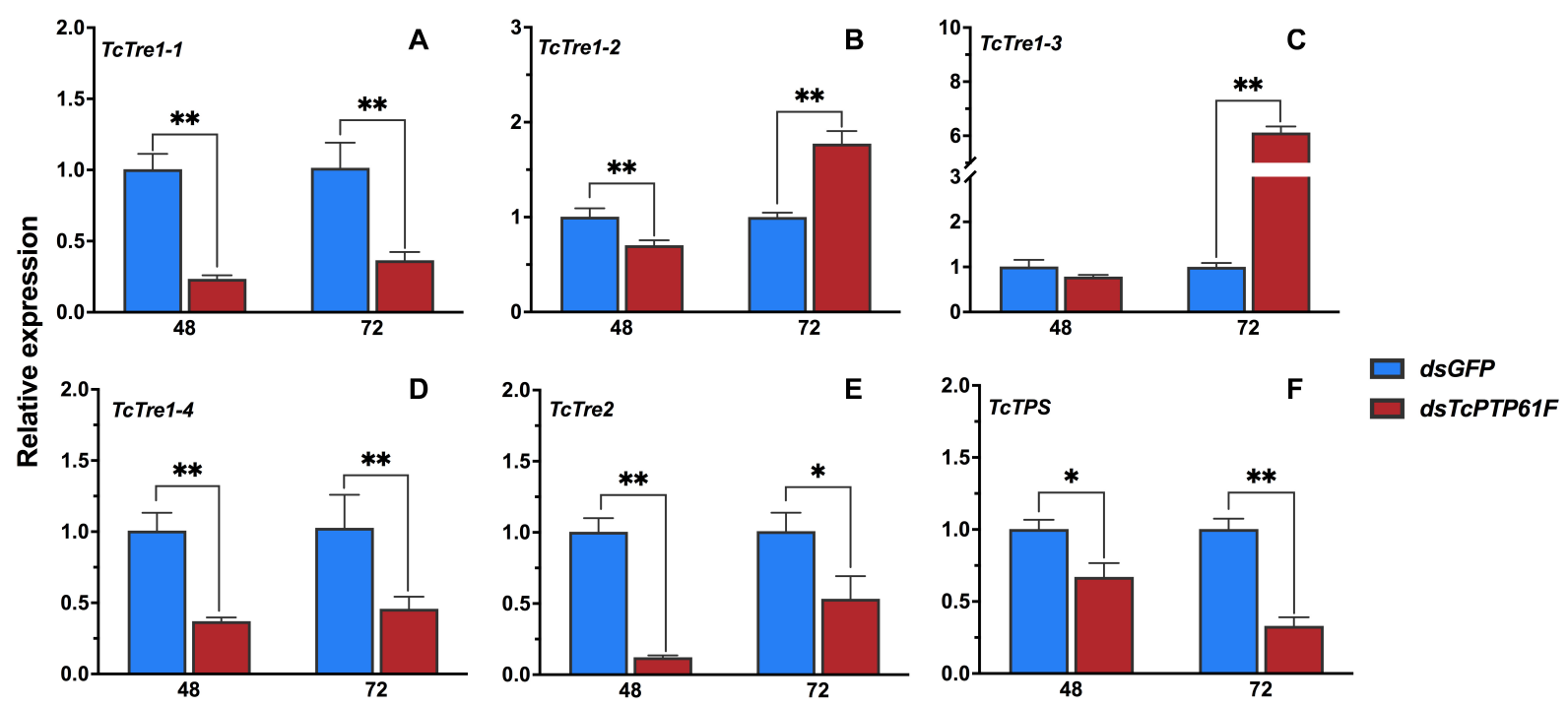

Hours after injection

FIGURE 3 | Effects of TCPTP61F knockdown on the expressions of six trehalose metabolic pathway genes. The relative expression levels of five trehalases (TCTre, A-E) and one trehalose-6-phosphate synthases (TCTPS, F) at 48 and $72 \mathrm{~h}$ after TCPTP61F or GFP dsRNA injection. The expression values were calculated by comparison to the dsGFP group, which was normalized at 1. Significant differences were identified by Student's $t$-test $\left({ }^{*} P<0.05\right.$, $\left.{ }^{* *} P<0.01\right)$.
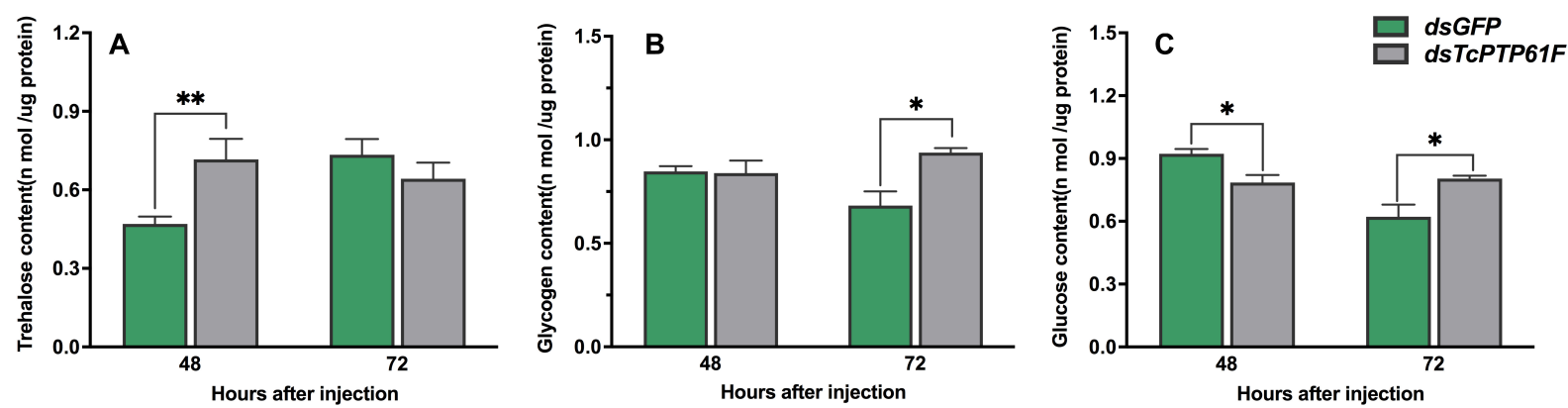

FIGURE 4 | Effects of TCPTP61F knockdown on content of trehalase (A), glycogen (B), and glucose (C). Significant differences were identified by Student's t-test $(* P<0.05, * * P<0.01)$.
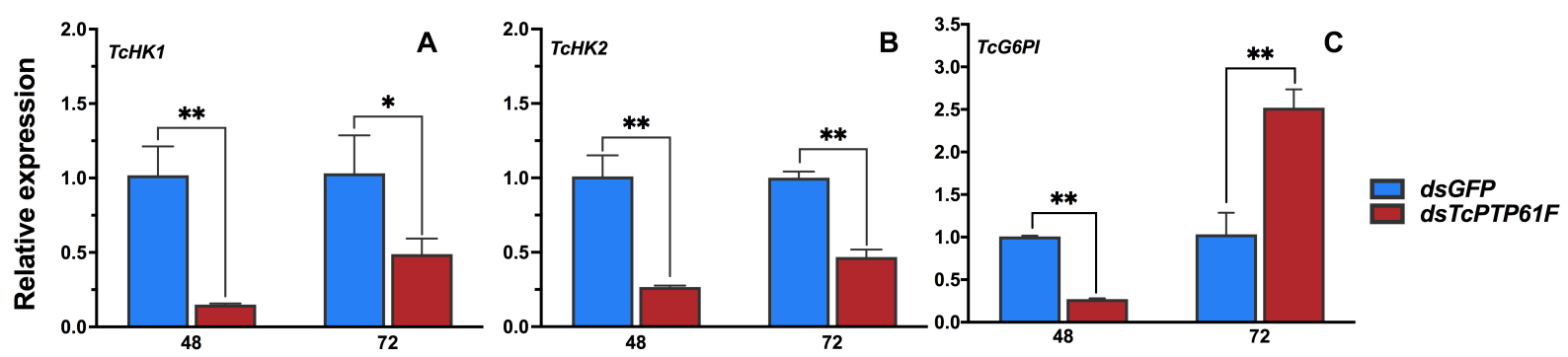

Hours after injection

FIGURE 5 | Effects of TCPTP61F knockdown on the expressions of three glycolytic pathway pathway genes. The relative expression levels of TCHK1 (A), TCHK2 (B) and TCG6PI (C) at 48 and $72 \mathrm{~h}$ after TCPTP61F or GFP dsRNA injection. Significant differences were identified by Student's $t$-test $\left({ }^{*} P<0.05,{ }^{* *} P<0.01\right)$.

development, metamorphosis, stress recovery, chitin synthesis, and flight (Wegener et al., 2003, 2010; Tatun et al., 2014; Shukla et al., 2015; Tang et al., 2017; Zhang et al., 2017). In addition, trehalase is critical to the role of trehalose in insect physiology because it is required for regulation of metabolism and glucose generation (Shukla et al., 2015). Therefore, we tested the gene 

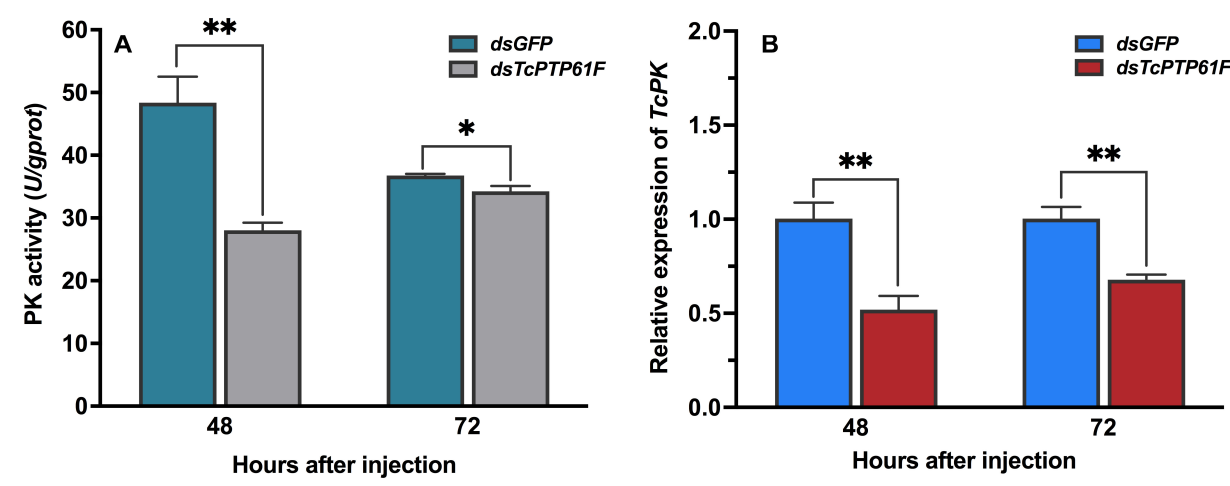

FIGURE 6 | Effect of TCPTP61F knockdown on pyruvate kinase activity (A) and expression of TCPK gene (B). Significant differences were identified by Student's t-test $\left({ }^{\star} P<0.05,{ }^{* \star} P<0.01\right)$.

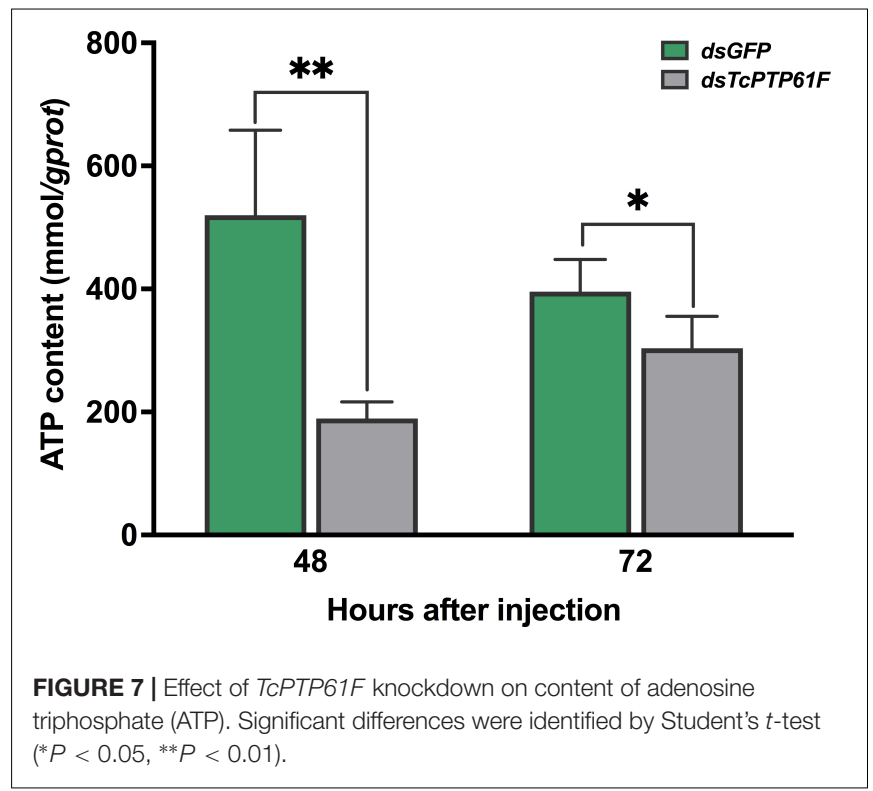

expression of the trehalose metabolism pathway of T. castaneum after RNAi and found that almost all Tre and TPS genes were significantly downregulated. However, TcTre1-2 and TcTre1-3 increased at $72 \mathrm{~h}$ after injection. These results suggest that the inhibition of TCPTP61F effected the regulation of the trehalose metabolism pathway. To further analyze its effect on trehalose metabolism, we tested the trehalose content and found that the synthesis of trehalose increased significantly at $48 \mathrm{~h}$ and returned to normal levels at $72 \mathrm{~h}$ (Figure 4A). This finding may be the result of the conversion of these three kinds of sugars. From the results of glucose and glycogen, we observed that trehalose accumulated at $48 \mathrm{~h}$ and was converted into glucose and glycogen after $72 \mathrm{~h}$, resulting in a significant increase in glycogen and glucose content at $72 \mathrm{~h}$ (Figures 4B,C). The increased expression of TcTre1-2 and TcTre1-3 might be main cause of the destruction of glucose hemostasis. These results all indicate that the silencing of TcPTP61F can have an effect on trehalose metabolism.
Glycolysis is an important method of energy metabolism and it involves the process of glucose decomposed into pyruvate, under an anaerobic environment, by a series of enzymes (Sunna et al., 1997). Among these enzymes, hexokinase (HK) is the first rate-limiting enzyme in the glycolytic pathway, which plays a key role in glucose homeostasis and energy metabolism through glucose (Glc) phosphorylation and Glc signaling (Ge et al., 2019). Therefore, we tested the expression of $H K$ to investigate the effect of dsTcPTP61F injection on the glucose homeostasis and energy metabolism of $T$. castaneum. The results showed that the expression of two TcHK genes in T. castaneum was significantly downregulated (Figures $\mathbf{5 A}, \mathbf{B}$ ). However, the glucose content decreases significantly at $48 \mathrm{~h}$, it may be due to less glucose being synthesized. Furthermore, the glucose content increased significantly after RNAi at $72 \mathrm{~h}$, which may be caused by the decomposition of a large amount of trehalose.

The PK enzyme catalyzes the conversion of phosphoenolpyruvate and ADP to pyruvate and ATP in glycolysis, and pyruvate can be further converted into acetylCoA that can combine with oxaloacetic acid to enter the TCA cycle (Israelsen and Vander Heiden, 2015). The TCA cycle, together with the subsequent electron transport chain, is one of the main metabolic pathways that provides energy to support cellular homeostasis under aerobic conditions (Gaster et al., 2012). Our results showed that PK enzyme activity was significantly reduced after TcPTP61F knockdown. Adenosine triphosphate has a fundamental intracellular role and is the direct energy source of most activities in living cells (Bodin and Burnstock, 2001). In this study, after TcPTP61F knockdown, the ATP content was significantly decreased both at 48 and $72 \mathrm{~h}$.

Based on our results and previous studies, we propose a hypothesis for the roles of TCPTP61F in regulating energy metabolism of T. castaneum (Figure 8). PTP61F acts as insulin receptor inhibitor that causes changes in the distribution of trehalose, glucose, and glycogen as well as a decreased glycolysis level. Downregulation of TCPTP61F caused a decreased ATP content. The inhibition of TCPTP61F resulted in disorders of energy metabolism in $T$. castaneum. 


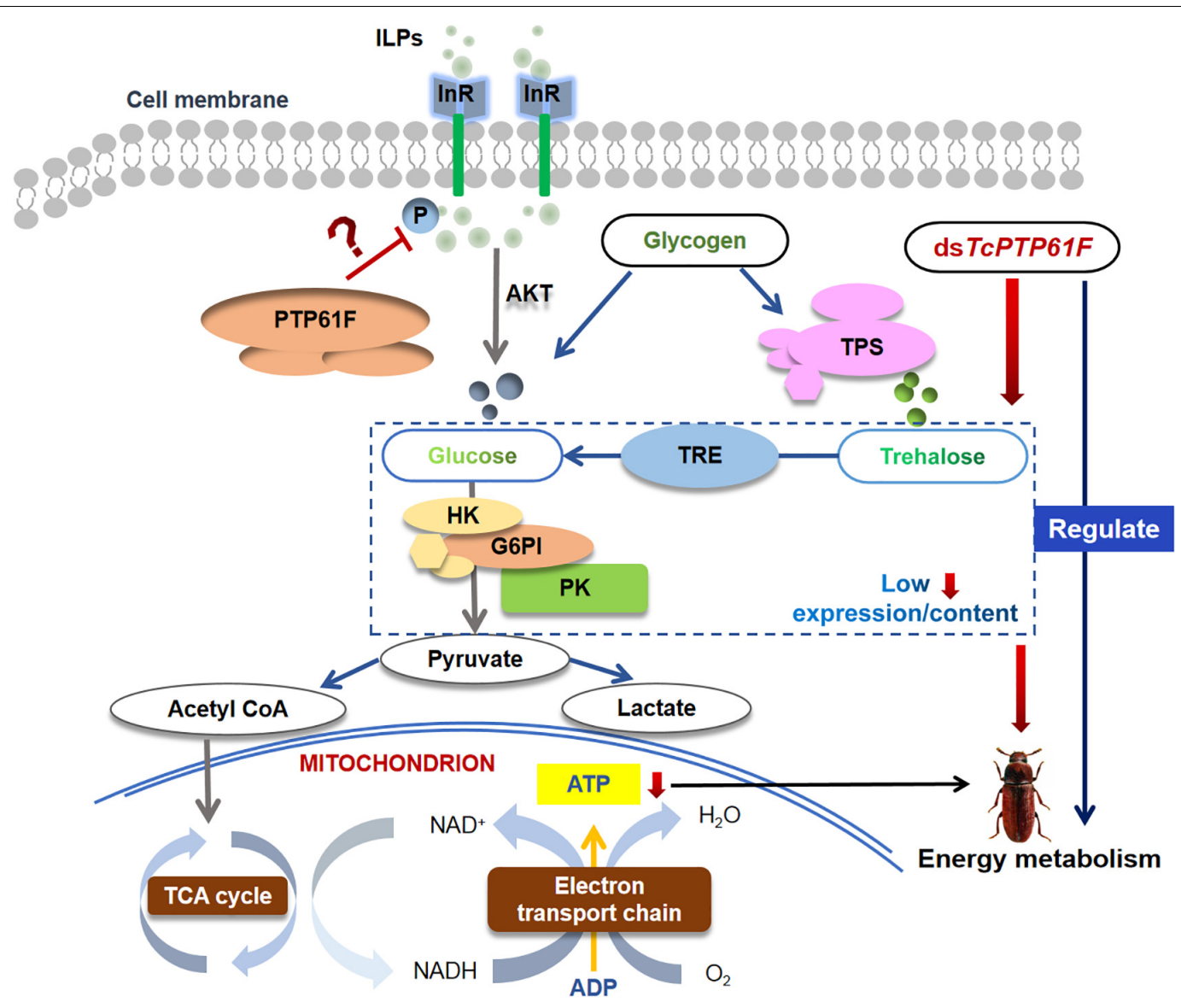

FIGURE 8 | Schematic description of the hypothesis for the roles of TCPTP61F in regulating energy metabolism of T. castaneum. PTP61F acts as insulin receptor inhibitor causes changes in the distribution of trehalose, glucose, and glycogen as well as a decreased glycolysis level. Downregulation of TCPTP61F caused a decreased ATP content. The inhibition of TCPTP61F resulted in disorders of energy metabolism in T. castaneum.

\section{DATA AVAILABILITY STATEMENT}

The raw data supporting the conclusions of this article will be made available by the authors, without undue reservation, to any qualified researcher.

\section{AUTHOR CONTRIBUTIONS}

$\mathrm{K}-\mathrm{KX}, \mathrm{B}-\mathrm{YP}$, and CL conceived and designed the experiments, and wrote the manuscript. B-YP, Y-YW, and Q-QR performed the experiments. CL revised the manuscript. All authors gave final approval for the publication. All authors contributed to the article and approved the submitted version.

\section{FUNDING}

This study was supported by the National Natural Science Foundation of China (31960542), the Discipline and Master's Site Construction Project of Guiyang University by Guiyang City Financial Support Guiyang University (SH-2020), the Program for Science and Technology Youth Talents in Department of Education in Guizhou Province (2018298), the Training Program for High Level Innovative Talents of Guizhou Province (20164020), and the Program for First-Class Discipline Construction in Guizhou Province (201785).

\section{SUPPLEMENTARY MATERIAL}

The Supplementary Material for this article can be found online at: $\quad$ https://www.frontiersin.org/articles/10.3389/fphys.2020. 01071/full\#supplementary-material

FIGURE S1 | Multiple alignments of the amino acid sequence of TCPTP61F with homologs from other insect species. Identical and similar amino acids are marked with the same color. Gaps have been introduced to permit alignment. The heights of the pink bars below the aligned sequences represent the degree of similarity of the amino acids. The red box indicate the conserved catalytic cysteine. TcPTP61F (Tribolium castaneum; GenBank accession number: XP_008190655.1),

DmPTP61F (Drosophila melanogaster; NP_476688.1), AgPTP61F1 (Anoplophora glabripennis; XP_018577682.1), DvPTP61F1 (Diabrotica virgifera; XP_028144768.1), LmPTPN1 (Locusta migratoria; AY150186.1), LdPTP61F1(Leptinotarsa decemlineata; XP_023013201.1), AtPTP61F1(Aethina tumida; XP_019879072.1), NvPTP61F1(Nicrophorus vespilloides; XP_017775944.1), and OtPTP61F1(Onthophagus taurus; XP_022904338.1). 
FIGURE S2 | Domain organization of PTP61Fs from T. castaneum and other insect speicies. The domain organization was generated with the SMART tool (http://smart.embl-heidelberg.de/) by using the protein sequences. The dark blue frame indicate the protein tyrosine coiled-coiloscioled-coliatase, catalytic domain

\section{REFERENCES}

Abaskharoun, M., Bellemare, M., Lau, E., and Margolis, R. U. (2010). Glypican-1, phosphacan/receptor protein-tyrosine phosphatase- $\zeta / \beta$ and its ligand, tenascin$\mathrm{C}$, are expressed by neural stem cells and neural cells derived from embryonic stem cells. ASN Neuro. 2:e0039. doi: 10.1042/AN20100001

Agouni, A., Tual-Chalot, S., Chalopin, M., Duluc, L., Mody, N., Martinez, C., et al. (2014). Hepatic protein tyrosine phosphatase 1B (PTP1B) deficiency protects against obesity-induced endothelial dysfunction. Biochem. Pharmacol. 92, 607-617. doi: 10.1016/j.bcp.2014.10.008

Alho, I., Costa, L., Bicho, M., and Coelho, C. (2013). The role of low-molecularweight protein tyrosine phosphatase (LMW-PTP ACP1) in oncogenesis Tumor. Biol. 34, 1979-1989. doi: 10.1007/s13277-013-0784-1

Alonso, A., Sasin, J., Bottini, N., Ilan, F., Iddo, F., Andrei, O., et al. (2004). Protein tyrosine phosphatases in the human genome. Cell 117, 699-711. doi: 10.1016/j. cell.2004.05.018

Bao, Y. Y., Xue, J., Wu, W. J., Wang, Y., Lv, Z. Y., and Zhang, C. X. (2011). An immune-induced reeler protein is involved in the Bombyx mori melanization cascade. Insect. Biochem. Mol. Biol. 41, 696-706. doi: 10.1016/j.ibmb.2011.05 001

Barraza, A., and Sánchez, F. (2013). Trehalases: a neglected carbon metabolism regulator? Plant Signal. Behav. 8:e24778. doi: 10.4161/psb.24778

Baum, J. A., Bogaert, T., Clinton, W., Heck, G. R., Feldmann, P., Ilagan, O., et al. (2007). Control of coleopteran insect pests through RNA interference. Nat. Biotechnol. 25, 1322-1326. doi: 10.1038/nbt1359

Biddinger, S. B., and Kahn, C. R. (2006). From mice to men: insights into the insulin resistance syndromes. Annu. Rev. Physiol. 68, 123-158. doi: 10.1146/annurev. physiol.68.040104.124723

Boateng, B. A., and Obengofori, D. (2008). “Major stored product arthropod pests," in Post-Harvest Science and Technology, eds E. W. Cornelius, D. Obeng-Ofori (Accra: Smartline Publishing Limited), 67-91.

Bodin, P., and Burnstock, G. (2001). Purinergic signalling: ATP release. Neurochem. Res. 26, 959-969. doi: 10.1023/a:1012388618693

Chen, J. X., Lyu, Z. H., Wang, C.Y., Cheng, J., and Lin, T. (2018). RNA interference of a trehalose-6-phosphate synthase gene reveals its roles in the biosynthesis of chitin and lipids in Heortia vitessoides (Lepidoptera: Crambidae). Insect Sci. 27, 212-223. doi: 10.1111/1744-7917.12650

Chen, Q. W., Jin, S., Zhang, L., Shen, Q. D., Wei, P., Wei, C. M., et al (2018). Regulatory functions of trehalose-6-phosphate synthase in the chitin biosynthesis pathway in Tribolium castaneum (Coleoptera: Tenebrionidae) revealed by RNA interference. Bull. Entomol. Res. 108, 388-399. doi: 10.1017/ S000748531700089X

Czech, M. P., and Corvera, S. (1999). Signaling mechanisms that regulate glucose transport. J. Biol. Chem. 274, 1865-1868. doi: 10.1074/jbc.274.4.1865

Ding, D., Liu, G. J., Hou, L., Gui, W. Y., Chen, B., and Kang, L. (2018) Genetic variation in PTPN1 contributes to metabolic adaptation to highaltitude hypoxia in Tibetan migratory locusts. Nat. Commun. 26:4991. doi: 10.1038/s41467-018-07529-8

Elbein, A. D., Pan, Y. T., Pastuszak, I., and Carroll, D. (2003). New insights on trehalose: a multifunctional molecule. Glycobiology 13, 17R-27R. doi: 10.1093/ glycob/cwg047

Forsell, P. A., Boie, Y., Montalibet, J., Collins, S., and Kennedy, B. P. (2000). Genomic characterization of the human and mouse protein tyrosine phosphatase-1B genes. Gene 260, 145-153. doi: 10.1016/s0378-1119(00) 00464-9

Gaster, M., Nehlin, J. O., and Minet, A. D. (2012). Impaired TCA cycle flux in mitochondria in skeletal muscle from type 2 diabetic subjects: marker or maker of the diabetic phenotype? Arch. Physiol. Biochem. 118, 156-189. doi: 10.3109/ 13813455.2012.656653

Ge, L. Q., Gu, H. T., Li, X., Zheng, S., Zhou, Z., Miao, H., et al. (2019). Silencing of triazophos-induced Hexokinase-1-like reduces fecundity in Nilaparvata lugens
(PTPC), and the light blue frame indicates the amino acid responsible for the recognition of Y-coiled-coiloscioled-coliatase. Structure of the coiled-coil and transmembrane region of PTP61Fs are represented as yellow ellipse and green polygon, respectively.

(Stål) (Hemiptera: Delphacidae). Pestic. Biochem. Physiol. 153, 176-184. doi: 10.1016/j.pestbp.2018.11.016

Hendriks, W. J. A. J., Elson, A., Harroch, S., Pulido, R., Stoker, A., and den Hertog, J. (2013). Protein tyrosine phosphatases in health and disease. FEBS J. 280, 708-730. doi: $10.1111 /$ febs. 12000

Hu, D., Luo, W., Fan, L. F., Liu, F. L., Gu, J., Deng, H. M., et al. (2016). Dynamics and regulation of glycolysis-tricarboxylic acid metabolism in the midgut of Spodoptera litura during metamorphosis. Insect. Mol. Biol. 25, 153-162. doi: $10.1111 /$ imb. 12208

Israelsen, W. J., and Vander Heiden, M. G. (2015). Pyruvate kinase: function, regulation and role in cancer. Semin. Cell Dev. Biol. 43, 43-51. doi: 10.1016/j. semcdb.2015.08.004

Jablonka, W., Senna, R., Nahu, T., Ventura, G., Menezes, L., and Silva-Neto, M. A. (2011). A transient increase in total head phosphotyrosine levels is observed upon the emergence of Aedes aegypti from the pupal stage. Mem. Inst. Oswaldo Cruz 106:546-552. doi: 10.1590/s0074-02762011000500005

Kim, Y., and Hong, Y. (2015). Regulation of hemolymph trehalose level by an insulin-like peptide through diel feeding rhythm of the beet armyworm, Spodoptera exigua. Peptides 68, 91-98. doi: 10.1016/j.peptides.2015.02.003

Klaman, L. D., Boss, O., Peroni, O. D., Kim, J. K., Martino, J. L., Zabolotny, J. M. et al. (2000). Increased energy expenditure, decreased adiposity, and tissuespecific insulin sensitivity in protein-tyrosine phosphatase 1B-deficient mice. Mol. Cell. Biol. 20, 5479-5489. doi: 10.1128/mcb.20.15.5479-5489.2000

Leevers, S. J. (2001). Growth control: invertebrate insulin surprises! Curr. Biol. 11, R209-R212. doi: 10.1016/s0960-9822(01)00107-5

Leyva, A., Quintana, A., Sánchez, M., Rodríguez, E. N., Cremata, J., and Sánchez, J. C. (2008). Rapid and sensitive anthrone-sulfuric acid assay in microplate format to quantify carbohydrate in biopharmaceutical products: method devlopment and validation. Biologicals 36, 134-141. doi: 10.1016/j.biologicals. 2007.09.001

Livak, K. J., and Schmittgen, T. D. (2001). Analysis of relative gene expression data using real-time quantitative PCR and the $2^{-\Delta \Delta}$ CT method. Methods 25, 402-408. doi: 10.1006/meth.2001.1262

Lochhead, P. A., Coghlan, M., Rice, S. Q., and Sutherland, C. (2001). Inhibition of GSK-3 selectively reduces glucose-6-phosphatase and phosphatase and phosphoenolypyruvate carboxykinase gene expression. Diabetes 50, 937-946. doi: 10.2337/diabetes.50.5.937

Loh, K., Merry, T. L., Galic, S., Wu, B. J., Watt, M. J., Zhang, S., et al. (2012). $\mathrm{T}$ cell protein tyrosine phosphatase (TCPTP) deficiency in muscle does not alter insulin signalling and glucose homeostasis. Diabetologia 55, 468-478. doi: 10.1007/s00125-011-2386-z

Lorenzen, M. D., Kimzey, T., Shippy, T. D., Brown, S. J., Denell, R. E., and Beeman, R. W. (2007). PiggyBac-based insertional mutagenesis in Tribolium castaneum using donor/helper hybrids. Insect Mol. Biol. 16, 265-275. doi: 10.1111/j.13652583.2007.00727.x

Mehmood, K., Husain, M., Aslam, M., Ahmedani, M. S., Aulahk, A. M., and Shaheen, F. A. (2018). Changes in the nutritional composition of maize flour due to Tribolium castaneum infestation and application of carbon dioxide to manage this pest. Environ. Sci. Pollut. Res. 25, 18540-18547. doi: 10.1007/ s11356-018-2063-6

Miyasaka, H., and Li, S. S. (1992). The cDNA cloning, nucleotide sequence and expression of an intracellular protein tyrosine phosphatase from mouse testis. Biochem. Biophys. Res. Commun. 185, 818-825. doi: 10.1016/0006-291x(92) 91700-z

Moretti, D. M., Ahuja, L. G., Nunes, R. D., Cudischevitch, C. O., Daumas-Filho, C. R. O., Medeiros-Castro, P., et al. (2014). Molecular analysis of Aedes aegypti classical protein tyrosine phosphatases uncovers an ortholog of mammalian PTP-1B implicated in the control of egg production in mosquitoes. PLoS One 9:e104878. doi: 10.1371/journal.pone.0104878

Mustelin, T., Vang, T., and Bottini, N. (2005). Protein tyrosine phosphatases and the immune response. Nat. Rev. Immunol. 5, 43-57. doi: 10.1038/nri1530 
Noh, M. Y., Beeman, R. W., and Arakane, Y. (2012). RNAi-based functional genomics in Tribolium castaneum and possible application for controlling insect pests. Entomol. Res. 42, 1-10. doi: 10.1111/j.1748-5967.2011.00437.x

Perkin, L. C., and Oppert, B. (2019). Gene expression in Tribolium castaneum life stages: identifying a species-specific target for pest control applications. Peer J. 7:e6946. doi: 10.7717/peerj.6946

Pimentel, M. A. G., Faroni, L. R. D. A., da Silva, F. H., Basista, M. D., and Guedes, R. N. (2010). Spread of phosphine resistance among brazilian populations of three species of stored product insects. Neotrop. Entomol. 39, 101-107. doi: 10.1590/s1519-566x2010000100014

Predel, R., Neupert, S., Garczynski, S. F., Crim, J. W., Brown, M. R., Russell, W. K., et al. (2010). Neuropeptidomics of the mosquito Aedes aegypti. J. Proteome. Res. 9, 2006-2015. doi: 10.1021/pr901187p

Prentice, K., Christiaens, O., Pertry, I., Bailey, A., Niblett, C., Ghislain, M., et al. (2017). RNAi-based gene silencing through dsRNA injection or ingestion against the African sweet potato weevil Cylas puncticollis (Coleoptera: Brentidae). Pest Manag. Sci. 73, 44-52. doi: 10.1002/ps.4337

Pri-Tal, B. M., Brown, J. M., and Riehle, M. A. (2008). Identification and characterization of the catalytic subunit of phosphatidylinositol 3-kinase in the yellow fever mosquito Aedes aegypti. Insect Biochem. Mol. Biol. 38, 932-939. doi: 10.1016/j.ibmb.2008.07.004

Riehle, M. A., and Brown, M. R. (2003). Molecular analysis of the serine/threonine kinase Akt and its expression in the mosquito Aedes aegypti. Insect Mol. Biol. 12, 225-232. doi: 10.1046/j.1365-2583.2003.00405.x

Sacco, F., Perfetto, L., Castagnoli, L., Cesareni, G. (2012). The human phosphatase interactome: an intricate family portrait. FEBS Lett. 586, 2732-2739. doi: 10. 1016/j.febslet.2012.05.008

Saltiel, A. R., and Kahn, C. R. (2001). Insulin signalling and the regulation of glucose and lipid metabolism. Nature 414, 799-806. doi: 10.1038/414799a

Satake, S., Masumura, M., Ishizaki, H., Nagata, K., Kataoka, H., Suzuki, A., et al. (1997). Bombyxin, an insulin-related peptide of insects, reduces the major storage carbohydrates in the silk worm Bombyx mori. Comp. Biochem. Physiol. B Biochem. Mol. Biol. 118, 349-357. doi: 10.1016/s0305-0491(97)00166-1

Shukla, E., Thorat, L. J., Nath, B. B., and Gaikwad, S. M. (2015). Insect trehalase: physiological significance and potential applications. Glycobiology 25, 357-367. doi: $10.1093 /$ glycob/cwu125

Sunna, A., Moracci, M., Rossi, M., and Antranikian, G. (1997). Glycosyl hydrolases from hyperthermophiles. Extremophiles 1, 27-13. doi: 10.1007/s007920050009

Tamang, A. M., Kalra, B., and Parkash, R. (2017). Cold and desiccation stress induced changes in the accumulation and utilization of proline and trehalose in seasonal populations of Drosophila immigrans. Comp. Biochem. Physiol. A Mol. Integr. Physiol. 203, 304-313. doi: 10.1016/j.cbpa.2016.10.011

Tang, B., Wei, P., Zhao, L. N., Shi, Z. K., Shen, Q. D., Yang, M. M., et al. (2016). Knockdown of five trehalase genes using RNA interference regulates the gene expression of the chitin biosynthesis pathway in Tribolium castaneum. BMC Biotechnol. 6:67. doi: 10.1186/s12896-016-0297-2

Tang, B., Yang, M. M., Shen, Q. D., Xu, Y. X., Wang, H. J., and Wang, S. G. (2017). Suppressing the activity of trehalase with validamycin disrupts the trehalose and chitin biosynthesis pathways in the rice brown planthopper, Nilaparvata lugens. Pestic. Biochem. Physiol. 137, 81-90. doi: 10.1016/j.pestbp.2016.10.003

Tatun, N., Wangsantitham, O.,Tungjitwitayakul, J., and Sakurai, S. (2014) Trehalase activity in fungus-growing termite, Odontotermes feae (Isoptera:
Termitideae) and inhibitory effect of validamycin. J. Econ. Entomol. 107, 1224 1232. doi: 10.1603/ec14051

Tian, L., Guo, E. E., Wang, S., Liu, S. M., Jiang, R. J., Cao, Y., et al. (2010). Developmental regulation of glycolysis by 20-hydroxyecdysone and juvenile hormone in fat body tissues of the silkworm, Bombyx mori. J. Mol. Cell Biol. 2, 255-263. doi: 10.1093/jmcb/mjq020

Tiganis, T., and Bennett, A. M. (2007). Protein tyrosine phosphatase function: the substrate perspective. Biochem. J. 402, 1-15. doi: 10.1042/BJ20061548

Tonks, N. K. (2006). Protein tyrosine phosphatases: from genes, to function, to disease. Nat. Rev. Mol. Cell Biol. 7, 833-846. doi: 10.1038/nrm2039

Tsou, R. C., and Bence, K. K. (2013). Central regulation of metabolism by protein tyrosine phosphatases. Front. Neurosci. 6:192. doi: 10.3389/fnins.2012.00192

Vital, W., Rezende, G. L., Abreu, L., Moraes, J., Lemos, F. J. A., Jr., et al. (2010). Germ band retraction as a landmark in glucose metabolism during Aedes aegypti embryogenesis. BMC Dev. Biol. 10, 25. doi: 10.1186/1471-213X-10-25

Wang, Y. J., He, H. W., Liu, L. N., Gao, C. Y., Xu, S., Zhao, P., et al. (2014). Inactivation and unfolding of protein tyrosine phosphatase from Thermus thermophilus HB27 during urea and guanidine hydrochloride denaturation. PLoS One 9:e107932. doi: 10.1371/journal.pone.0107932

Wegener, G., Macho, C., Schlöder, P., Kamp, G., and Ando, O. (2010). Long-term effects of the trehalase inhibitor trehazolin on trehalase activity in locust flight muscle. J. Exp. Biol. 213, 3852-3857. doi: 10.1242/jeb.042028

Wegener, G., Tschiedel, V., Schlöder, P., and Ando, O. (2003). The toxic and lethal effects of the trehalase inhibitor trehazolin in locusts are caused by hypoglycaemia. J. Exp. Biol. 206, 1233-1240. doi: 10.1242/jeb.00217

White, K. P., Rifkin, S. A., Hurban, P., and Hogness, D. S. (1999). Microarray analysis of Drosophila development during metamorphosis. Science 286, 2179 2184. doi: 10.1126/science.286.5447.2179

White, M. F. (1998). The IRS-signalling system: a network of docking proteins that mediate insulin action. Mol. Cell. Biochem. 182, 3-11.

Wu, C. L., Buszard, B., Teng, C. H., Chen, W. L., Warr, C. G., Tiganis, T., et al. (2011). Dock/Nck facilitates PTP61F/PTP1B regulation of insulin signalling. Biochem. J. 439, 151-159. doi: 10.1042/BJ20110799

Yasugi, T., Yamada, T., and Nishimura, T. (2017). Adaptation to dietary conditions by trehalose metabolism in Drosophila. Sci. Rep. 7:1619. doi: 10.1038/s41598017-01754-9

Zhang, L., Qiu, L. Y.,Yang, H. L., Wang, H. J., Zhou, M., Wang, S. G., et al. (2017). Study on the effect of wing bud chitin metabolism and its developmental network genes in the brown planthopper, Nilaparvata lugens, by knockdown of TRE gene. Front. Physiol. 8:750. doi: 10.3389/fphys.2017.00750

Conflict of Interest: The authors declare that the research was conducted in the absence of any commercial or financial relationships that could be construed as a potential conflict of interest.

Copyright (c) $2020 \mathrm{Xu}$, Pan, Wang, Ren and Li. This is an open-access article distributed under the terms of the Creative Commons Attribution License (CC BY). The use, distribution or reproduction in other forums is permitted, provided the original author(s) and the copyright owner(s) are credited and that the original publication in this journal is cited, in accordance with accepted academic practice. No use, distribution or reproduction is permitted which does not comply with these terms. 